\title{
Role of extracellular signal-regulated kinase 1/2 signaling underlying cardiac hypertrophy
}

\author{
Zhi-Peng Yan ${ }^{1,2}$, Jie-Ting $\mathrm{Li}^{2}$, Ni Zeng ${ }^{2}$, Guo-Xin $\mathrm{Ni}^{1}$ \\ ${ }^{1}$ School of Sport Medicine and Rehabilitation, Beijing Sport University, China \\ ${ }^{2}$ Department of Rehabilitation Medicine, The First Affiliated Hospital of Fujian Medical University, China
}

\begin{abstract}
Cardiac hypertrophy is the result of increased myocardial cell size responding to an increased workload and developmental signals. These extrinsic and intrinsic stimuli as key drivers of cardiac hypertrophy have spurred efforts to target their associated signaling pathways. The extracellular signal-regulated kinases 1/2 (ERK1/2), as an essential member of mitogen-activated protein kinases (MAPKs), has been widely recognized for promoting cardiac growth. Several modified transgenic mouse models have been generated through either affecting the upstream kinase to change ERK1/2 activity, manipulating the direct role of ERK1/2 in the heart, or targeting phosphatases or MAPK scaffold proteins to alter total ERK1/2 activity in response to an increased workload. Using these models, both regulation of the upstream events and modulation of each isoform and indirect effector could provide important insights into how ERK1/2 modulates cardiomyocyte biology. Furthermore, a plethora of compounds, inhibitors, and regulators have emerged in consideration of ERK, or its MAPK kinases, are possible therapeutic targets against cardiac hypertrophic diseases. Herein, is a review of the available evidence regarding the exact role of ERK1/2 in regulating cardiac hypertrophy and a discussion of pharmacological strategy for treatment of cardiac hypertrophy. (Cardiol J 2021; 28, 3: 473-482)
\end{abstract}

Key words: ERK1/2, cardiac hypertrophy, cardiomyocyte, genetic approaches, pharmacological strategy

\section{Introduction}

The mammalian heart is a muscular pump that circulates blood throughout the body to maintain perfusion of peripheral organs, which meets their demand during both regular and stressful conditions. In response to an increased workload, enlargement of the heart occurs and is defined as an increase in heart size without changes in myocyte number. Physiological hypertrophy observed in normal growth or trained athletes is considered an adaptive and compensatory response to maintain cardiac function and improved cardiac contractility. By contrast, cardiac hypertrophy under pathological conditions, such as ischemic heart disease, hypertension, and heart failure, is referred to as pathological hypertrophy. This type of hypertrophy is associated with the production of high levels of hemodynamic overload, interstitial fibrosis, and myocardial cell damage and loss [1,2]. Because cardiac hypertrophy plays a central role in cardiac remodeling and is an independent risk factor for cardiac events, understanding the molecular mechanisms is vital.

Previous studies have shown that a group of medical genetic syndromes referred to as RASopathie are caused by germline mutations in genes that encode components or regulators of the RAS-RAF-MEK-ERK pathway, which include neurofibromatosis type 1 , Noonan syndrome, Costello syndrome, and cardio-facio-cutaneous syndrome [3]. These patients suffered from cardiomyopathies suggesting that the RAS/MAPK pathway is critical to normal heart development. Moreover, before mouse genetics entered the mainstream of experiments, studies were conducted mainly on

Address for correspondence: Prof. Guo-Xin Ni, School of Sports Medicine and Rehabilitation, Beijing Sport University, tel: +(86)-10-62989780, fax:+(86)-10-62989670, e-mail: niguoxin@bsu.edu.cn 
cultured neonatal rat cardiomyocytes or inadequate "myocyte-like" cell lines which discovered the activation of the extracellular signal-regulated kinases $1 / 2(\mathrm{ERK} 1 / 2)$ pathway under the hormone and mechanical stretch in the heart, suggesting a direct causation between ERK1/2 signaling and the hypertrophic response [4,5]. To support this hypothesis, the use of dominant negative MEK1, antisense oligonucleotides against ERK1/2, dephosphorylation of ERK1/2, and pharmacologic inhibitors of MEK1/2 provided convincing data, indicating that MEK1-ERK1/2 is both necessary and sufficient for cardiac hypertrophy [6-10]. Autophosphorylation of ERK1/2 on Thr188 was also observed in isolated cardiomyocytes induced by hypertrophic stimulus [11]. Although considerable evidence elucidates that the activated MEK1/2-ERK1/2 can regulate cardiac hypertrophy in vitro, the results of other similarly designed culture-based studies are quite different $[12,13]$; one study even suggested that ERK1/2 activation is anti-hypertrophic [14]. In addition to traditional two-dimensional (2D) in vitro systems, three-dimensional (3D) tissue models have also offered new tools in the study of cardiovascular disease recently [15]. In response to $3 \mathrm{D}$ conditions, the activation of ERK1/2 was observed during cardiomyogenesis, and the phosphorylation of ERK1/2 was higher compared to cells on 2D films, which provides insight into ERK1/2 pathways driving heart development $[16,17]$. With regard to the whole organ phenomenon in a dynamically changing neuroendocrine environment under cardiac hypertrophy and heart failure, culture-based or tissue-engineering approaches have only provided some basic physiological parameters within a largely $2 \mathrm{D}$ or $3 \mathrm{D}$ environment [18]. However, these approaches will always be necessary because of the simplified process established in a largely isolated system and temporal relationships. More recently, a new method, known as mathematical optimization framework, also analyzed the complex ERK1/2 cascade in cardiomyocytes to find efficient adjustment screws for this cascade that is important for cardiomyocyte survival and maladaptive heart muscle growth [19].

In this review, the focus was on the main thread of the RAS-RAF-MEK-ERK pathway and summarize the more recent knowledge about the functional activity of ERK pathway in the cardiac organ to reveal the exact role of ERK1/2 in regulating cardiac hypertrophy. Also, the latest scientific results of addressing ERK signaling as a therapeutic target for the treatment of cardiac hypertrophy is discussed.

\section{Components and regulation of the ERK cascade}

As ERK1 and ERK2 are $83 \%$ identical in sequence and share most of the same signaling activities, they are usually referred to as ERK1/2 [20]. However, these two proteins are not entirely functionally redundant. For instance, ERK1 knockout animals appear normal and are viable, while ERK2 deletion results in embryonic lethality [21]. In cardiomyocytes, the canonical ERK1/2 signal cascade is initiated by the activation of the small $\mathrm{G}$ protein rat sarcoma (RAS) in the cell membrane, which leads to the recruitment and activation of RAF-1 (MAP3K), which further phosphorylates the dual-specificity protein kinases MEK1/2 (MAP2K); eventually, MEK1/2 characteristically activate ERK1/2 (MAPK) by phosphorylating the threonine-glutamate-tyrosine (TEY) motif in the phosphorylation loop. Active ERK is then released from the MEK and phosphorylates a wide array of cytoplasmic substrates. Alternatively, activation of ERK is translocated to the nucleus and phosphorylates numerous transcription factors, which result in the induction of growth and proliferation and in the prevention of cell death [22]. Furthermore, the specificity of ERK biological effects is influenced by many factors, including (i) duration and strength of the signals, (ii) interaction with various scaffold proteins, (iii) subcellular localization, (iv) extensive cross-talk and interplay between the ERK cascade and other intracellular signaling pathways, and (v) presence of several similar isoforms at each tier of the cascade [23]. Although many of these mechanisms could independently determine signaling specificity and magnitude of the signaling outcome, they often work in coordination with each other to ensure proper downstream effects. Additionally, the prohypertrophic, pro-survival and pro-death effects of ERK1/2 converge on mitochondria upon their crucial roles in metabolism of cardiomyocyte. In response to types of stimuli, ERK1/2 can modulate mitochondria-mediated cardiomyocyte function directly through the interaction with mitochondria [24], or indirectly, by activation/inhibition of ERK-dependent downstream signaling molecules or mediators [25-28]. Finally, the inactivation of ERKs is regulated by various phosphatases, including dual-specificity MAPK phosphatases (MKPs), protein serine/threonine phosphatases (PPs), and protein tyrosine phosphatases (PTP) $[29,30]$. 


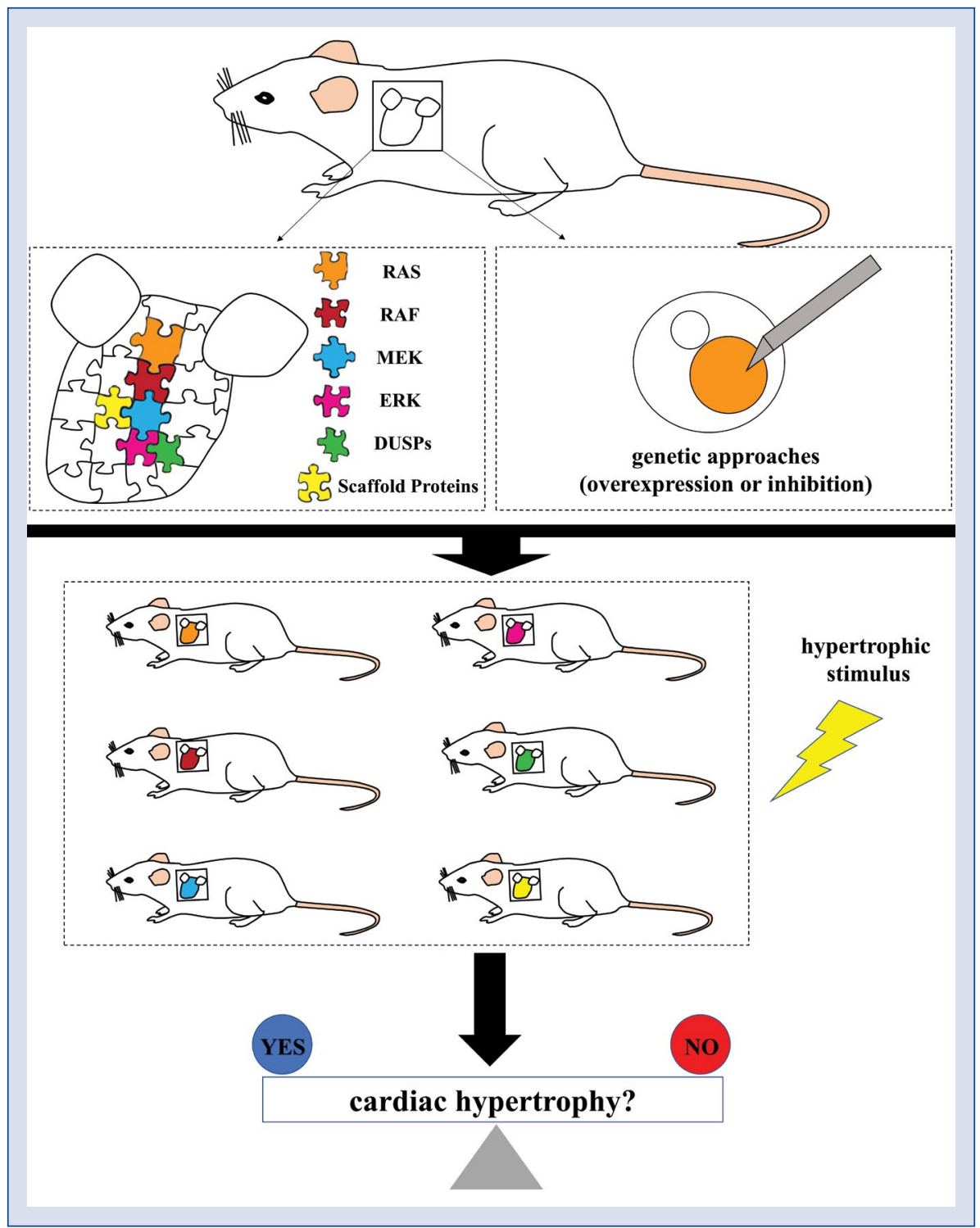

Figure 1. Schematic diagram of extracellular signal-regulated kinases (ERK) pathway and mitogen-activated protein kinase (MAPK)-associated genetic mouse models. In cardiomyocytes, the canonical ERK1/2 signal cascade is initiated by the activation of the small G protein rat sarcoma (RAS) in the cell membrane, which leads to the recruitment and activation of RAF-1, which further phosphorylates the dual-specificity protein kinases MEK1/2; eventually, MEK1/2 characteristically activate ERK1/2. In response to growth factors, hormones, and mechanical stress, the RAS-RAF-MEK-ERK pathway, dual-specificity phosphatases (DUSPs) and scaffold proteins were overexpressed or inhibited in the mouse to change ERK1/2 activity, which examines the connection between ERK activation and cardiac hypertrophy; RAF — rapidly accelerated fibrosarcoma; MEK — MAPK/ERK kinase.

\section{ERK1/2 and cardiac hypertrophy}

Studying the role of the RAS-RAF-MEK-ERK pathway in the heart, did not, herein, satisfy a detailed understanding of the physiological function of the cascade, which revealed that cardiomyocytes are isolated from their physiological environment, the present investigation focuses on the emergence of genetic approaches in mice using gained or lost functional mutants (Fig. 1).

\section{Targeting upstream kinase}

The previously published H-Ras-V12 transgenic model with overstimulating of the RAS hinted at the trend of decompensation and dilatation of the heart and developed a chain of events that contrib- 
uted to the pathogenesis of cardiovascular disease [31-33]. Subsequently, Wei et al. [34] demonstrated that discontinuing overactivation of this pathway after the onset of cardiomyopathy could lead to improved survival and cardiomyopathy lesion scores, suggesting the reversibility of early pathogenic hypertrophy. More recently, the possible anti-hypertrophic effect of RAS inhibition in the setting of pressure-overload cardiac hypertrophy in rats was examined [35]. After pressure-overload induction and Ras-mutant gene transfer, the hypertrophic degrees of the heart in both Ras mutants were similar. However, the Ras-Val12 mutant increases left ventricular systolic diameter and reduces left ventricular fractional shortening compared to control and dominant negative mutant N17-DNRas (DN-Ras). Moreover, DN-Ras exhibited some similarities with physiologic hypertrophy, especially the lower expression of markers of pathologic cardiac hypertrophy. Although these studies used different promoters to drive RAS expression, the same constitutively activated RAS mutants were generated, indicating that the inactivation of Ras is cardioprotective rather than anti-hypertrophic. Ras directly activates Raf-1, which may subsequently lead to MEK1-ERK1/2 activation. It also activates other intracellular signaling pathways. Importantly, its activation is involved in pathological changes in sarcoplasmic reticulum calcium handling [36, 37].

Activating mutations in the serine-threonine kinase Raf cause cardiac hypertrophy and contribute to Noonan syndrome in humans. Heterozygous Raf1L613V mice were generated and exhibited eccentric cardiac hypertrophy, aberrant cardiac fetal gene expression, and decompensation following pressure overload, but treatment of these mice with MEK inhibitors (PD0325901) or constitutive deletion of ERK effector (RSK3) rescued Rafmediated cardiac hypertrophy and other phenotypic abnormalities [38, 39]. In addition, a dominant negative form of Raf- 1 animals had obvious resistance to the development of cardiac hypertrophy and hypertrophic gene induction in response to pressure overload [40]. Both groups found that, while Raf-1 kinase activity was essential for cardiac hypertrophy, enhanced MEK-ERK activity was critical for causing RAF1-mutant phenotypes. However, recently, Yin et al. [41] showed that left ventricular hypertrophy was due to the interplay of cardiac cell types. Using inducible Raf1L613V expression, mutant RAF1 expression in cardiomyocytes enhanced $\mathrm{Ca} 2+$ sensitivity and cardiac contractility rather than hypertrophy. By contrast, endothelial/endocardial (EC)-restricted mutant expression does not affect contractility, but evokes hypertrophy. Moreover, aberrant RAF1 activity in cardiomyocytes or cardiac fibroblasts, but not ECs, contributes to pressure overload-induced fibrosis in Noonan syndrome cardiomyopathy. These results support a paradigm shift away from the myocyte-centric view of cardiac development and disease.

Even though RAS transgenic mice exhibited cardiac hypertrophy associated with cardiomyopathy, MEK1 transgenic mice showed a stable concentric hypertrophy without any signal of decompensation up to 12 months of age [42]. Furthermore, these mice expressing activated MEK1 showed a dramatic increase in cardiac function measured by echocardiography and isolated working heart preparation, and there was activation of ERK1/2, but not p38 or JNK. In addition, MEK1 transgenic mice showed resistance to ischemia/ /reperfusion-induced apoptosis. These outcomes suggest that MEK-ERK not only sufficiently induces normally hypertrophic response, but that it also has a bearing on partial resistance to apoptosis.

Taken together, these results support that the RAS-RAF-MEK-ERK pathway is generally regarded as pro-hypertrophic and suggest that sustained cascade activation also plays a cardioprotective role in the heart. Furthermore, the closer the upstream kinase is to ERK, the stronger the correlation with hypertrophic process. This may be because the initial upstream kinase is more susceptible to interference from other factors.

\section{Targeting ERK1/2}

In view that evidence of non-redundancy is apparent from isoform-specific ERK targeted mice, overexpression of intrinsically active ERK1 and ERK2 in the heart were generated to further test the effects of ERK1/2. A recent study reported transgenic mice expressing activated ERK1 under the transcriptional control of the $\alpha$-MHC promoter - which, similar to the observations in hypertrophy, is phosphorylated on both the TEY and the Thr207 motifs and is overexpressed at pathophysiological levels - developed a modest adaptive hypertrophy with increased contractile function and without fibrosis [43]. Nevertheless, another recent study demonstrated that volume overload-induced eccentric hypertrophy is associated with reduced cardiac ERK1/2 activation while phosphorylation of other MAPKs was unaffected in vivo. However, transgenic mice with cardiomyocyte-specific ERK2 overexpression did not alter left ventricular dilation and hypertrophy [44]. Importantly, Molkentin et al. 
[18] found that high levels of ERK2 overexpression in the heart from two independent transgenic lines with the same $\alpha$-MHC promoter did not induce hypertrophy. However, MEK1 transgenic mice crossed with ERK2 transgenic mice showed synergistic hypertrophy. Although ERK2 seems to be dominant in the results of the knockout experiment, the single overexpression of these two kinds of MAPK appears inconsistent, and the relationship between MEK1/2 and ERK2 is likely to be close. These results indicate that ERK1 may induce the hypertrophic effect after the inhibition of MEK1/2. Further, a novel ERK2 autophosphorylation site, other than TEY phosphorylation, was found on Thr188 after stimulation with pressure overload and in failing human hearts [11]. The equivalent phosphorylation was also discovered on EKR1 at Thr207 [45, 46]. The authors generated several lines of transgenic mice overexpressing ERK2 with mutations at Thr188. Compared with baseline wild type mice, no hypertrophy was observed in these mice [11]. After pressure overload, the ERK2T188D (gain-of-function ERK) mice showed more striking hypertrophy, not the ERK2T188A or ERK2T188S (phosphorylationdeficient ERK) mice. This mechanism depends on upstream signals - specifically, activation of Gqcoupled receptors, which release $\mathrm{G} \beta \gamma$; activation of the entire Raf-MEK-ERK cascade; subsequent phosphorylation of ERK1/2 within the TEY motif; and ERK dimerization. The integration of these signaling events leads to autophosphorylation of ERK2 at Thr188. In addition, the phosphorylation of Thr188 is related to the pathological morphology of hypertrophy [47]. It is generally believed that the specific role of Thr188 phosphorylation of ERK1/2 in vivo was to transform adaptive ERK signals into maladaptive signals. However, this notion is based on a study of mice overexpressing ERK2 Thr188 mutants, which may eliminate catalytic activity of ERK.

Contrary to the gain of function of ERK1/2, some reports on ERK knockout mice suggested that ERK1/2 signaling may not be necessary to mediate cardiac growth in vivo. ERK1 null (-/-) and ERK2 null (+/-) mice showed no reduction in cardiac hypertrophy response to pathologic stimulus-induced by transverse aortic constriction (TAC) or to physiologic stimulus-induced by swimming [48]. Moreover, mice lacking all ERK1/2 protein in the heart (ERK1-/- ERK2fl/fl-Cre) were generated. After eliminating both isoforms, the heart still increased in weight with both aging and pathological stress stimulation, where the heart showed spon- taneous dilatation and the cardiomyocytes showed spontaneous lengthening [49]. Another study examined the cardiomyocyte-specific deletion of the ERK2 gene (ERK2cko mice). Following short-term pathological hypertrophic stresses, the mutant mice showed attenuated hypertrophic remodeling characterized by a blunted increase in the cross-sectional area of individual myocytes. However, the absence of ERK2 did not affect physiological hypertrophy induced by exercise [50].

Above all, these results suggest that either ERK1/2 are not critical for mediating cardiac hypertrophy under pathophysiologic stress or the remaining ERK1/2 activity in each gene-targeted mouse model was sufficient to mediate the signaling events required to drive the hypertrophic response. This outcome places ERK at the crossroads of cardiac hypertrophic signaling pathways and raises the possibility that different isoforms of ERK may play different roles in regulating the growth of cardiac myocytes.

\section{Targeting phosphatases and MAPK scaffold proteins}

Due to the high embryonic lethality, it was difficult to generate ERK1-/- ERK2+/- mice (3 of 4 alleles deleted) to address the necessity of ERK1/2 signaling in mediating cardiac hypertrophy. Therefore, other approaches are desirable to more effectively alter total ERK1/2 activity within the heart. Dual-specificity phosphatases (DUSPs), the largest family of MAPK-selective phosphatases, act to dephosphorylate both the p-Ser/Thr and -Tyr residues that are essential for cytosolic and/ /or nuclear MAPK activity. A precedent for these phosphatases appeared with the description of DUSP6-/- mice [51]. An increase in basal ERK1/2 phosphorylation in the absence of DUSP6 was identified, but no effect was observed on other MAPKs after stimulation. DUSP6-/- mice with larger hearts was not due to hypertrophy, but rather to hypercellularity of the myocytes. A recent study confirmed a similar finding in the zebrafish heart by suppressing the Dusp6 function, which showed that DUSP6 attenuated Ras/MAPK signaling during regeneration, and inactivation of DUSP6 could enhance cardiac repair [52]. Opposite to the activation of ERK1/2, multiple lines of DUSP6 contained in the mouse heart were specifically generated [48]. Similar to ERK1 null (-/-) and ERK2 null (+/-) mice, low-, medium-, and high-Dusp6 Tg mice showed no reduction in hypertrophy after pressure overload stimulation, neuroendocrine agonist infusion, or physiologic exercise stimulation, though 
the activation of all cardiac ERK1/2 at baseline were nearly eliminated. Notably, a phosphatase known as DUSP8 has drawn attention recently. DUSP8-/- mice increased ERK1/2 phosphorylation and were mildly hypercontractile at baseline with the concentric remodeling of the heart, which provided prolonged protection from progressing towards heart failure in two surgery-induced disease models [53]. While cardiac-specific overexpression of DUSP8 produced spontaneous eccentric remodeling with heart failure, overexpression of DUSP8 in the heart caused dephosphorylation of all three major MAPK terminal effectors. Overall, although these studies suggest that ERK1/2 manipulated by DUSP6 and DUSP8 are not required for mediating hypertrophy per se, one critical point to consider is that other DUSPs such as DUSP2, $-4,-5,-7$, and -9 also dephosphorylate ERK1/2 [54]. While 13 DUSP proteins are dedicated to regulating and recycling the MAPKs, each appears to have a highly specialized regulatory role.

Given that MAPK scaffolding proteins enable the formation of specific signaling complexes and subcellular localization in the activation of an MAPK cascade, many studies of genetic mouse models, specifically MAPK scaffolding proteins, have further reinforced a direct role for ERK1/2 pathway in stress-induced cardiac hypertrophy. Scaffold proteins tether MAPK/ERK signaling at the sarcomere and plasma membrane in the cardiac muscle and regulate ERK signaling strength and duration [55]. As such, ANKRD1, which conducts the components of the sarcomere-associated biomechanical sensors, can be markedly induced by various hypertrophic stimuli and in distinct animal models of hypertrophy. ANKRD1-overexpressing transgenic mice developed less hypertrophy, and no differences were evident in heart function compared to wild-type mice in TAC- and isoproterenolinduced models [56], which were mediated by the inhibition of ERK1/2 and TGF- $\beta$ pathways. Conversely, striking cardiac hypertrophy with reactivation of the cardiac fetal gene program induced by chronic phenylephrine infusion in wild-type mice was completely eliminated in ANKRD1 null mice via ANKRD1-ERK-GATA4 complex to regulate hypertrophic responses [57]. This phenomenon may be caused by different hypertrophic stimuli. Respectively, IQGAP1, which bind to the plasma membrane, played a part in the maintenance of cardiomyocyte physiology and the induction of adaptive hypertrophy. IQGAP1-null mice initially developed compensatory hypertrophy and unaltered basal heart function in response to pressure overload, but with a prolonged stimulus, they showed acceleration towards the development of maladaptive hypertrophy resulting in a decrease of cardiac contractility [58]. These results emphasize that different scaffold proteins may mediate the spatial regulation of ERK1/2 activation, which in turn determines the substrate specificity of pathological states associated with cardiac hypertrophy. Additionally, MAPK scaffolds, such as MP1 [59] and DYRK1A [60], have been characterized in the heart, but their role in cardiac remodeling has not yet been addressed. By extension, readers can see several excellent recent reviews on this issue $[61,62]$.

\section{Pharmacological strategy for the treatment of cardiac hypertrophy}

Despite substantial advances, there is still a major demand for finding novel therapeutic strategies to use in the hypertrophic process of cardiac remodeling and cardiac events. Although ERK activation events need to be investigated further and refined in detail, ERK or its MAPK kinases, is a possible therapeutic target for a pharmacological strategy against cardiac hypertrophic diseases. If the intrinsic manipulation of ERK is insufficient to reliably achieve complete remission, an obvious alternative strategy is to target extrinsically potential compounds, inhibitors, or regulators.

\section{Compounds}

Recent works are underway to discover compounds that inhibit this pathway and thus provide a potential therapeutic agent that could attenuate cardiac growth. Traditional Chinese medicine has been reported to be effective for the treatment of cardiac hypertrophy in animal models, such as Baicalein and Bu-Shen-Jiang-Ya [63] decoction (BSJYD). Baicalein, known for its antibacterial, antiviral, and anti-inflammatory effects, protects against cardiac hypertrophy and fibrosis in response to chronic pressure overload by regulating the MEK-ERK1/2 signaling pathway [63]. Additionally, BSJYD treatment decreases systolic blood pressure and heart rate efficiently and suppresses the hypertension-induced cardiac hypertrophy associated with the suppressive effect of BSJYD on the EKR signaling pathway [64]. Other compounds were also reported to target the ERK pathway. Sun et al. [65] showed that gentisic acid attenuates pressure overload-induced cardiac hypertrophy and fibrosis in mice through inhibition of the ERK1/2 pathway. Additionally, Li et al. [66] demonstrated 
that selumetinib could attenuate pathological and physiological cardiac hypertrophy in vivo caused by pressure overload and swimming. In short, these preclinical studies encourage the emergence of more effective compounds to target cardiac hypertrophy with aberrant ERK activation.

\section{Inhibitors}

Developed for the treatment of various cancers, kinase inhibitors are also used to reduce excessive signal activation in cardiac hypertrophy. One study that explored the effects of the MEK inhibitor of Pimasertib on cardiac hypertrophy and heart failure showed a reversible effect of Pimasertib on a transgenic model expressing an activated MET receptor, which was attributed to the inhibition of ERK1/2 pathway [67]. Several in vitro studies have also explored the effects of MEK inhibitor effects on cultured cardiomyocytes. Two of the most commonly utilized are PD98059 and U0126. PD98059 reversed leukemia inhibitory factor-induced cardiomyocyte hypertrophy [68]. Similarly, U0126 blocked cardiomyocyte growth induced by endothelin-1 and phenylephrine [69]. As available MEK inhibitors for the ERK signaling pathway, these agents target regions distinct from the ATP pocket. However, these two inhibitors inhibit all MEKs upstream of the ERKs, including MEK1, MEK2, and MEK5. More recently, a newly potent and selective MEK inhibitor called TAK733 was confirmed to suppress norepinephrine or phenylepinephrine-induced cardiomyocyte hypertrophy through the depression of MEK-ERK signaling [70]. These studies support the role of MEK-ERK cascade in promoting hypertrophy, but the inherent non-selectivity of some inhibitors should not be ignored because of their effects on MEK5.

\section{Regulators}

It is common knowledge that MAPK pathways are largely associated with $\mathrm{G}$ protein-coupled receptor (GPCRs)-mediated signaling. Belonging to cell membrane receptors, GPCRs interact with $G$ proteins to activate multiple downstream intracellular cascades and in turn modulate subsets of effector proteins [71]. Regulators of $\mathrm{G}$ protein signaling (RGS) proteins are negative regulators of $G$ protein mediated signaling that serve as GTPase activating protein for heterotrimeric $\mathrm{G}$ proteins. Recently, accumulating studies have demonstrated that RGS proteins can regulate cardiac hypertrophy independent of GTPase-activating protein activity. Among them, RGS3 [72], RGS5 [73], RGS10 [74], RGS12 [75], and RGS14 [76] protect against pressure overload-induced hypertrophic response and improve cardiac function by inhibiting MEK-ERK1/2 signaling. Notably, unlike most overexpression approaches leading to beneficial effects on the heart, RGS12-defcient hearts showed a decreased cardiomyocyte cross area without predisposing the heart to adverse remodeling and failure. Although RGS10, RGS12 and RGS14 belong to the R12/D subfamily, the reason for such opposing responses is not completely understood. Considering the anti-hypertrophic effect attributed to some RGS proteins through MEK-ERK1/2 signaling, their potential as targets for therapeutic intervention warrants further examination.

\section{Conclusions}

In summary, although the ERK cascade is a major signaling component and regulates many distinct cellular processes, the functional role of the ERK1/2 in the heart still presents a potential dilemma. As this review shows, many current approaches are based solely on opposite ways to dissect the cardiac disease states, such as cell culture versus mouse genetics, direct versus indirect manipulation of ERK1/2, and gain versus loss of function. However, the ERK cascade is mainly regulated at several levels by different phosphatases and scaffold proteins, other signaling pathways, subcellular localization, and even different isoforms per se. Many of these mechanisms can work together to determine the final specificity of the cascade. While uncontrolled activation of RAS-RAF-MEK-ERK signaling may lead to cardiac hypertrophy, inhibiting the pathway may also make the heart more susceptible to stress induced cardiomyocyte death. Therefore, to unravel the ERK1/2 mechanisms, more sophisticated model systems are required, which will help us to make sure the critical role of ERK1/2 and the ensuing effects in the heart. In light of the lack of uniform results in ERK biology in cardiac hypertrophy, translation of the knowledge about pro-hypertrophic signaling pathways may lead to both exciting and challenging clinical insights.

\section{Acknowledgements}

This work was supported by National Natural Science Foundation of China (81871848).

Conflict of interest: None declared 


\section{References}

1. Shimizu I, Minamino T. Physiological and pathological cardiac hypertrophy. J Mol Cell Cardiol. 2016; 97: 245-262, doi: 10.1016/j. yjmcc.2016.06.001, indexed in Pubmed: 27262674.

2. Nakamura M, Sadoshima J. Mechanisms of physiological and pathological cardiac hypertrophy. Nat Rev Cardiol. 2018; 15(7): 387-407, doi: 10.1038/s41569-018-0007-y, indexed in Pubmed: 29674714.

3. Rauen KA. The RASopathies. Annu Rev Genomics Hum Genet. 2013; 14: 355-369, doi: 10.1146/annurev-genom-091212-153523, indexed in Pubmed: 23875798.

4. Post GR, Goldstein D, Thuerauf DJ, et al. Dissociation of $\mathrm{p} 44$ and p42 mitogen-activated protein kinase activation from receptorinduced hypertrophy in neonatal rat ventricular myocytes. J Biol Chem. 1996; 271(14): 8452-8457, doi: 10.1074/jbc.271.14.8452, indexed in Pubmed: 8626545 .

5. Yamazaki T, Tobe K, Hoh E, et al. Mechanical loading activates mitogen-activated protein kinase and $\mathrm{S} 6$ peptide kinase in cultured rat cardiac myocytes. J Biol Chem. 1993; 268(16): 12069-12076, indexed in Pubmed: 7685031.

6. Ueyama T, Kawashima S, Sakoda T, et al. Requirement of activation of the extracellular signal-regulated kinase cascade in myocardial cell hypertrophy. J Mol Cell Cardiol. 2000; 32(6): 947-960, doi: 10.1006/jmcc.2000.1135, indexed in Pubmed: 10888249.

7. Liang F, Lu S, Gardner DG. Endothelin-dependent and -independent components of strain-activated brain natriuretic peptide gene transcription require extracellular signal regulated kinase and p38 mitogen-activated protein kinase. Hypertension. 2000; 35(1 Pt 2): 188-192, doi: 10.1161/01.hyp.35.1.188, indexed in Pubmed: 10642296.

8. Liang Q, Wiese RJ, Bueno OF, et al. The transcription factor GATA4 is activated by extracellular signal-regulated kinase 1- and 2-mediated phosphorylation of serine 105 in cardiomyocytes. Mol Cell Biol. 2001; 21(21): 7460-7469, doi: 10.1128/ MCB.21.21.7460-7469.2001, indexed in Pubmed: 11585926.

9. Glennon PE, Kaddoura S, Sale EM, et al. Depletion of mitogenactivated protein kinase using an antisense oligodeoxynucleotide approach downregulates the phenylephrine-induced hypertrophic response in rat cardiac myocytes. Circ Res. 1996; 78(6): 954-961, doi: 10.1161/01.res.78.6.954, indexed in Pubmed: 8635245.

10. Ferguson BS, Harrison BC, Jeong MY, et al. Signal-dependent repression of DUSP5 by class I HDACs controls nuclear ERK activity and cardiomyocyte hypertrophy. Proc Natl Acad Sci U S A. 2013; 110(24): 9806-9811, doi: 10.1073/pnas.1301509110, indexed in Pubmed: 23720316.

11. Lorenz K, Schmitt JP, Schmitteckert EM, et al. A new type of ERK1/2 autophosphorylation causes cardiac hypertrophy. Nat Med. 2009; 15(1): 75-83, doi: 10.1038/nm.1893, indexed in Pubmed: 19060905.

12. Thorburn J, Frost JA, Thorburn A. Mitogen-activated protein kinases mediate changes in gene expression, but not cytoskeletal organization associated with cardiac muscle cell hypertrophy. J Cell Biol. 1994; 126(6): 1565-1572, doi: 10.1083/jcb.126.6.1565, indexed in Pubmed: 8089186.

13. Thorburn J, McMahon M, Thorburn A. Raf- 1 kinase activity is necessary and sufficient for gene expression changes but not sufficient for cellular morphology changes associated with cardiac myocyte hypertrophy. J Biol Chem. 1994; 269(48): 30580-30586, indexed in Pubmed: 7982977.
14. Silberbach M, Gorenc T, Hershberger RE, et al. Extracellular signal-regulated protein kinase activation is required for the anti-hypertrophic effect of atrial natriuretic factor in neonatal rat ventricular myocytes. J Biol Chem. 1999; 274(35): 24858-24864, doi: 10.1074/jbc.274.35.24858, indexed in Pubmed: 10455158.

15. Mathur A, Ma Z, Loskill P, et al. In vitro cardiac tissue models: Current status and future prospects. Adv Drug Deliv Rev. 2016; 96: 203-213, doi: 10.1016/j.addr.2015.09.011, indexed in Pubmed: 26428618.

16. Vozzi F, Logrand F, Cabiati M, et al. Biomimetic engineering of the cardiac tissue through processing, functionalization, and biological characterization of polyester urethanes. Biomed Mater. 2018; 13(5): 055006, doi: 10.1088/1748-605X/aaca5b, indexed in Pubmed: 29869614.

17. Ghosh LD, Jain A, Sundaresan NR, et al. Elucidating molecular events underlying topography mediated cardiomyogenesis of stem cells on 3D nanofibrous scaffolds. Mater Sci Eng C Mater Biol Appl. 2018; 88: 104-114, doi: 10.1016/j.msec.2018.03.012, indexed in Pubmed: 29636125.

18. Molkentin JD, Robbins J. With great power comes great responsibility: using mouse genetics to study cardiac hypertrophy and failure. J Mol Cell Cardiol. 2009; 46(2): 130-136, doi: 10.1016/j. yjmcc.2008.09.002, indexed in Pubmed: 18845155.

19. Breitenbach T, Lorenz K, Dandekar T. How to steer and control ERK and the ERK signaling cascade exemplified by looking at cardiac insufficiency. Int J Mol Sci. 2019; 20(9), doi: 10.3390/ ijms20092179, indexed in Pubmed: 31052520.

20. Lloyd AC. Distinct functions for ERKs? J Biol. 2006; 5(5): 13, doi: 10.1186/jbiol46, indexed in Pubmed: 16879721.

21. Gerits N, Kostenko S, Moens U. In vivo functions of mitogenactivated protein kinases: conclusions from knock-in and knockout mice. Transgenic Res. 2007; 16(3): 281-314, doi: 10.1007/ s11248-006-9052-0, indexed in Pubmed: 17219248.

22. Wang Y. Mitogen-activated protein kinases in heart development and diseases. Circulation. 2007; 116(12): 1413-1423, doi: 10.1161/ /CIRCULATIONAHA.106.679589, indexed in Pubmed: 17875982.

23. Shaul YD, Seger R. The MEK/ERK cascade: from signaling specificity to diverse functions. Biochim Biophys Acta. 2007; 1773(8): 1213-1226, doi: 10.1016/j.bbamcr.2006.10.005, indexed in Pubmed: 17112607.

24. García-Niño WR, Correa F, Rodríguez-Barrena JI, et al. Cardioprotective kinase signaling to subsarcolemmal and interfibrillar mitochondria is mediated by caveolar structures. Basic Res Cardiol. 2017; 112(2): 15, doi: 10.1007/s00395-017-0607-4, indexed in Pubmed: 28160133.

25. Nomura S, Satoh M, Fujita T, et al. Cardiomyocyte gene programs encoding morphological and functional signatures in cardiac hypertrophy and failure. Nat Commun. 2018; 9(1): 4435, doi: 10.1038/s41467-018-06639-7, indexed in Pubmed: 30375404.

26. Lu YT, Li LZ, Yang YL, et al. Succinate induces aberrant mitochondrial fission in cardiomyocytes through GPR91 signaling. Cell Death Dis. 2018; 9(6): 672, doi: 10.1038/s41419-018-0708-5, indexed in Pubmed: 29867110.

27. Huang CY, Chen JY, Kuo CH, et al. Mitochondrial ROS-induced ERK1/2 activation and HSF2-mediated AT R upregulation are required for doxorubicin-induced cardiotoxicity. J Cell Physiol. 2018; 233(1): 463-475, doi: 10.1002/jcp.25905, indexed in Pubmed: 28295305.

28. Gartz M, Darlington A, Afzal MZ, et al. Exosomes exert cardioprotection in dystrophin-deficient cardiomyocytes via ERK1/2- 
p38/MAPK signaling. Sci Rep. 2018; 8(1): 16519, doi: 10.1038/ s41598-018-34879-6, indexed in Pubmed: 30410044.

29. Liu R, Molkentin JD. Regulation of cardiac hypertrophy and remodeling through the dual-specificity MAPK phosphatases (DUSPs). J Mol Cell Cardiol. 2016; 101: 44-49, doi: 10.1016/j. yjmcc.2016.08.018, indexed in Pubmed: 27575022.

30. Junttila MR, Li SP, Westermarck J. Phosphatase-mediated crosstalk between MAPK signaling pathways in the regulation of cell survival. FASEB J. 2008; 22(4): 954-965, doi: 10.1096/fj.06-7859rev, indexed in Pubmed: 18039929.

31. Hunter JJ, Tanaka N, Rockman HA, et al. Ventricular expression of a MLC-2v-ras fusion gene induces cardiac hypertrophy and selective diastolic dysfunction in transgenic mice. J Biol Chem. 1995; 270(39): 23173-23178, doi: 10.1074/jbc.270.39.23173, indexed in Pubmed: 7559464.

32. Zheng M, Dilly K, Dos Santos Cruz J, et al. Sarcoplasmic reticulum calcium defect in Ras-induced hypertrophic cardiomyopathy heart. Am J Physiol Heart Circ Physiol. 2004; 286(1): H424-H433, doi: 10.1152/ajpheart.00110.2003, indexed in Pubmed: 12969887.

33. Mitchell S, Ota A, Foster W, et al. Distinct gene expression profiles in adult mouse heart following targeted MAP kinase activation. Physiol Genomics. 2006; 25(1): 50-59, doi: 10.1152/ physiolgenomics.00224.2005, indexed in Pubmed: 16368875.

34. Wei BR, Martin PL, Hoover SB, et al. Capacity for resolution of Ras-MAPK-initiated early pathogenic myocardial hypertrophy modeled in mice. Comp Med. 2011; 61(2): 109-118, indexed in Pubmed: 21535921.

35. Ramos-Kuri M, Rapti K, Mehel H, et al. Dominant negative Ras attenuates pathological ventricular remodeling in pressure overload cardiac hypertrophy. Biochim Biophys Acta. 2015; 1853(11 Pt A): 2870-2884, doi: 10.1016/j.bbamcr.2015.08.006, indexed in Pubmed: 26260012.

36. Ho PD, Zechner DK, He H, et al. The Raf-MEK-ERK cascade represents a common pathway for alteration of intracellular calcium by Ras and protein kinase $\mathrm{C}$ in cardiac myocytes. J Biol Chem. 1998; 273(34): 21730-21735, doi: 10.1074/jbc.273.34.21730, indexed in Pubmed: 9705309.

37. Rose BA, Force T, Wang Y. Mitogen-activated protein kinase signaling in the heart: angels versus demons in a heart-breaking tale. Physiol Rev. 2010; 90(4): 1507-1546, doi: 10.1152/ physrev.00054.2009, indexed in Pubmed: 20959622.

38. Wu X, Simpson J, Hong JH, et al. MEK-ERK pathway modulation ameliorates disease phenotypes in a mouse model of Noonan syndrome associated with the Raf1(L613V) mutation. J Clin Invest. 2011; 121(3): 1009-1025, doi: 10.1172/JCI44929, indexed in Pubmed: 21339642.

39. Passariello CL, Martinez EC, Thakur H, et al. RSK3 is required for concentric myocyte hypertrophy in an activated Raf1 model for Noonan syndrome. J Mol Cell Cardiol. 2016; 93: 98-105, doi: 10.1016/j.yjmcc.2016.02.020, indexed in Pubmed: 26940993.

40. Harris IS, Zhang S, Treskov I, et al. Raf-1 kinase is required for cardiac hypertrophy and cardiomyocyte survival in response to pressure overload. Circulation. 2004; 110(6): 718-723, doi: 10.1161/01.CIR.0000138190.50127.6A, indexed in Pubmed: 15289381.

41. Yin JC, Platt MJ, Tian X, et al. Cellular interplay via cytokine hierarchy causes pathological cardiac hypertrophy in RAF1-mutant Noonan syndrome. Nat Commun. 2017; 8: 15518, doi: 10.1038/ ncomms15518, indexed in Pubmed: 28548091.
42. Bueno OF, De Windt LJ, Tymitz KM, et al. The MEK1-ERK1/2 signaling pathway promotes compensated cardiac hypertrophy in transgenic mice. EMBO J. 2000; 19(23): 6341-6350, doi: 10.1093/emboj/19.23.6341, indexed in Pubmed: 11101507.

43. Mutlak M, Schlesinger-Laufer M, Haas T, et al. Extracellular signal-regulated kinase (ERK) activation preserves cardiac function in pressure overload induced hypertrophy. Int J Cardiol. 2018; 270: 204-213, doi: 10.1016/j.ijcard.2018.05.068, indexed in Pubmed: 29857938.

44. Jochmann S, Elkenani M, Mohamed BA, et al. Assessing the role of extracellular signal-regulated kinases 1 and 2 in volume overload-induced cardiac remodelling. ESC Heart Fail. 2019; 6(5): 1015-1026, doi: 10.1002/ehf2.12497, indexed in Pubmed: 31322843.

45. Smorodinsky-Atias K, Goshen-Lago T, Goldberg-Carp A, et al. Intrinsically active variants of Erk oncogenically transform cells and disclose unexpected autophosphorylation capability that is independent of TEY phosphorylation. Mol Biol Cell. 2016; 27(6): 1026-1039, doi: 10.1091/mbc.E15-07-0521, indexed in Pubmed: 26658610.

46. Lai S, Pelech S. Regulatory roles of conserved phosphorylation sites in the activation T-loop of the MAP kinase ERK1. Mol Biol Cell. 2016; 27(6): 1040-1050, doi: 10.1091/mbc.E15-07-0527, indexed in Pubmed: 26823016.

47. Ruppert C, Deiss K, Herrmann S, et al. Interference with ERK(Thr188) phosphorylation impairs pathological but not physiological cardiac hypertrophy. Proc Natl Acad Sci USA. 2013; 110(18): 7440-7445, doi: 10.1073/pnas.1221999110, indexed in Pubmed: 23589880 .

48. Purcell NH, Wilkins BJ, York A, et al. Genetic inhibition of cardiac ERK1/2 promotes stress-induced apoptosis and heart failure but has no effect on hypertrophy in vivo. Proc Natl Acad Sci USA. 2007; 104(35): 14074-14079, doi: 10.1073/pnas.0610906104, indexed in Pubmed: 17709754.

49. Kehat I, Davis J, Tiburcy M, et al. Extracellular signal-regulated kinases 1 and 2 regulate the balance between eccentric and concentric cardiac growth. Circ Res. 2011; 108(2): 176-183, doi: 10.1161/ CIRCRESAHA.110.231514, indexed in Pubmed: 21127295.

50. Ulm S, Liu W, Zi M, et al. Targeted deletion of ERK2 in cardiomyocytes attenuates hypertrophic response but provokes pathological stress induced cardiac dysfunction. J Mol Cell Cardiol. 2014; 72: 104-116, doi: 10.1016/j.yjmcc.2014.03.002, indexed in Pubmed: 24631771.

51. Maillet M, Purcell NH, Sargent MA, et al. DUSP6 (MKP3) null mice show enhanced ERK1/2 phosphorylation at baseline and increased myocyte proliferation in the heart affecting disease susceptibility. J Biol Chem. 2008; 283(45): 31246-31255, doi: 10.1074/jbc.M806085200, indexed in Pubmed: 18753132.

52. Missinato MA, Saydmohammed M, Zuppo DA, et al. Dusp6 attenuates Ras/MAPK signaling to limit zebrafish heart regeneration. Development. 2018; 145(5), doi: 10.1242/dev.157206, indexed in Pubmed: 29444893.

53. Liu R, van Berlo JH, York AJ, et al. DUSP8 regulates cardiac ventricular remodeling by altering ERK1/2 signaling. Circ Res. 2016; 119(2): 249-260, doi: 10.1161/CIRCRESAHA.115.308238, indexed in Pubmed: 27225478.

54. Owens DM, Keyse SM. Differential regulation of MAP kinase signalling by dual-specificity protein phosphatases. Oncogene. 2007; 26(22): 3203-3213, doi: 10.1038/sj.onc.1210412, indexed in Pubmed: 17496916. 
55. Casar B, Crespo P. ERK signals: scaffolding scaffolds? Front Cell Dev Biol. 2016; 4: 49, doi: 10.3389/fcell.2016.00049, indexed in Pubmed: 27303664.

56. Song Y, Xu J, Li Y, et al. Cardiac ankyrin repeat protein attenuates cardiac hypertrophy by inhibition of ERK1/2 and TGF- $\beta$ signaling pathways. PLoS One. 2012; 7(12): e50436, doi: 10.1371/journal. pone.0050436, indexed in Pubmed: 23227174.

57. Zhong L, Chiusa M, Cadar AG, et al. Targeted inhibition of ANKRD1 disrupts sarcomeric ERK-GATA4 signal transduction and abrogates phenylephrine-induced cardiomyocyte hypertrophy. Cardiovasc Res. 2015; 106(2): 261-271, doi: 10.1093/cvr/cvv108, indexed in Pubmed: 25770146.

58. Sbroggiò M, Carnevale D, Bertero A, et al. IQGAP1 regulates ERK1/2 and AKT signalling in the heart and sustains functional remodelling upon pressure overload. Cardiovasc Res. 2011; 91(3): 456-464, doi: 10.1093/cvr/cvr103, indexed in Pubmed: 21493702.

59. Kolch W. Coordinating ERK/MAPK signalling through scaffolds and inhibitors. Nat Rev Mol Cell Biol. 2005; 6(11): 827-837, doi: 10.1038/nrm1743, indexed in Pubmed: 16227978.

60. Himpel S, Tegge W, Frank R, et al. Specificity determinants of substrate recognition by the protein kinase DYRK1A. J Biol Chem. 2000; 275(4): 2431-2438, doi: 10.1074/jbc.275.4.2431, indexed in Pubmed: 10644696.

61. Liang Y, Sheikh F. Scaffold proteins regulating extracellular regulated kinase function in cardiac hypertrophy and disease. Front Pharmacol. 2016; 7: 37, doi: 10.3389/fphar.2016.00037, indexed in Pubmed: 26973524.

62. Tarone G, Sbroggiò M, Brancaccio M. Key role of ERK1/2 molecular scaffolds in heart pathology. Cell Mol Life Sci. 2013; 70(21): 4047-4054, doi: 10.1007/s00018-013-1321-5, indexed in Pubmed: 23532408.

63. Zong J, Zhang Dp, Zhou H, et al. Baicalein protects against cardiac hypertrophy through blocking MEK-ERK1/2 signaling. J Cell Biochem. 2013; 114(5): 1058-1065, doi: 10.1002/jcb.24445, indexed in Pubmed: 23225340.

64. Xiong X, Yang X, Duan L, et al. Traditional Chinese medicine suppresses left ventricular hypertrophy by targeting extracellular signal-regulated kinases signaling pathway in spontaneously hypertensive rats. Sci Rep. 2017; 7: 42965, doi: 10.1038/ srep42965, indexed in Pubmed: 28225023.

65. Sun S, Kee HJ, Jin Li, et al. Gentisic acid attenuates pressure overload-induced cardiac hypertrophy and fibrosis in mice through inhibition of the ERK1/2 pathway. J Cell Mol Med. 2018; 22(12): 5964-5977, doi: 10.1111/jcmm.13869, indexed in Pubmed: 30256522

66. Li C, Chen Z, Yang H, et al. Selumetinib, an Oral Anti-Neoplastic Drug, May Attenuate Cardiac Hypertrophy via Targeting the
ERK Pathway. PLoS One. 2016; 11(7): e0159079, doi: 10.1371/ journal.pone.0159079, indexed in Pubmed: 27438013.

67. Sala V, Gallo S, Gatti S, et al. Cardiac concentric hypertrophy promoted by activated Met receptor is mitigated in vivo by inhibition of Erk1,2 signalling with Pimasertib. J Mol Cell Cardiol. 2016; 93: 84-97, doi: 10.1016/j.yjmcc.2016.02.017, indexed in Pubmed: 26924269.

68. Kodama H, Fukuda K, Pan J, et al. Significance of ERK cascade compared with JAK/STAT and PI3-K pathway in gp130-mediated cardiac hypertrophy. Am J Physiol Heart Circ Physiol. 2000; 279(4): H1635-H1644, doi: 10.1152/ajpheart.2000.279.4.H1635, indexed in Pubmed: 11009450.

69. Yue TL, Gu JL, Wang C, et al. Extracellular signal-regulated kinase plays an essential role in hypertrophic agonists, endothelin-1 and phenylephrine-induced cardiomyocyte hypertrophy. J Biol Chem. 2000; 275(48): 37895-37901, doi: 10.1074/jbc. M007037200, indexed in Pubmed: 10984495.

70. Lee CY, Lee J, Seo HH, et al. TAK733 attenuates adrenergic receptor-mediated cardiomyocyte hypertrophy via inhibiting Erk-Thr188 phosphorylation. Clin Hemorheol Microcirc. 2019; 72(2): 179-187, doi: 10.3233/CH-180476, indexed in Pubmed: 30714951.

71. Woodard GE, Jardín I, Berna-Erro A, et al. Regulators of G-protein-signaling proteins: negative modulators of $\mathrm{G}$-protein-coupled receptor signaling. Int Rev Cell Mol Biol. 2015; 317: 97-183, doi: 10.1016/bs.ircmb.2015.02.001, indexed in Pubmed: 26008785.

72. Liu Yu, Huang He, Zhang Y, et al. Regulator of G protein signaling 3 protects against cardiac hypertrophy in mice. J Cell Biochem. 2014; 115(5): 977-986, doi: 10.1002/jcb.24741, indexed in Pubmed: 24375609.

73. Li H, He C, Feng J, et al. Regulator of G protein signaling 5 protects against cardiac hypertrophy and fibrosis during biomechanical stress of pressure overload. Proc Natl Acad Sci USA. 2010; 107(31): 13818-13823, doi: 10.1073/pnas.1008397107, indexed in Pubmed: 20643937.

74. Miao R, Lu Y, Xing X, et al. Regulator of G-protein signaling 10 negatively regulates cardiac remodeling by blocking mitogenactivated protein kinase-extracellular signal-regulated protein kinase 1/2 signaling. Hypertension. 2016; 67(1): 86-98, doi: 10.1161/HYPERTENSIONAHA.115.05957, indexed in Pubmed: 26573707.

75. Huang J, Chen L, Yao Y, et al. Pivotal role of regulator of G-protein signaling 12 in pathological cardiac hypertrophy. Hypertension. 2016; 67(6): 1228-1236, doi: 10.1161/HYPERTENSIONAHA.115.06877, indexed in Pubmed: 27091895.

76. Li Y, Tang XH, Li XH, et al. Regulator of $\mathrm{G}$ protein signalling 14 attenuates cardiac remodelling through the MEK-ERK1/2 signalling pathway. Basic Res Cardiol. 2016; 111(4): 47, doi: 10.1007/s00395-016-0566-1, indexed in Pubmed: 27298141. 